\title{
ERRATUM
}

Francesco Arena - Sergio Baldari

Antonio Centorrino - Maria Pia Calabrò

Giovanni Pajno · Salvatore Arena - Filippo Andò

Biagio Zuccarello • Giuseppe Romeo

\section{Mid- and long-term effects on pulmonary perfusion, anatomy and diaphragmatic motility in survivors of congenital diaphragmatic hernia}

Published online: 6 January 2006

(c) Springer-Verlag 2006

\section{Pediatr Surg Int (2005): DOI 10.007/s00383-005-1557-8}

In the original publication the name of the author Giovanni Pajno was rendered incorrectly.

The online version of the original article can be found at http:// dx.doi.org/10.1007/s00383-005-1557-8

F. Arena $(\bowtie) \cdot$ A. Centorrino $\cdot$ S. Arena $\cdot$ B. Zuccarello

G. Romeo

Dipartimento di Scienze Pediatriche Mediche e Chirurgiche U.O.C.

di Chirurgia Pediatrica, Università degli Studi di Messina,

Viale Gazzi A.O.U. Policlinico, Pad. NI, 98125 Messina, Italy

E-mail: arenaf@unime.it

Tel.: + 39-090-2213001

Fax: +39-090-2213010

S. Baldari

Dipartimento di Scienze Radiologiche U.O.C. di Medicina

Nucleare, Università degli Studi di Messina,

Viale Gazzi A.O.U. Policlinico, Pad. E,

98125 Messina, Italy

M. P. Calabrò

Dipartimento di Scienze Pediatriche Mediche e Chirurgiche U.O. di Cardiologia Pediatrica, Università degli Studi di Messina, Viale

Gazzi A.O.U. Policlinico, Pad. NI, 98125 Messina, Italy

G. Pajno

Dipartimento di Scienze Pediatriche Mediche e Chirurgiche U.O. di Allergologia Pediatrica, Università degli Studi di Messina,

Viale Gazzi A.O.U. Policlinico, Pad. NI, 98125 Messina, Italy

F. Andò

Dipartimento di Scienze Cardiovascolari e Toraciche U.O. C. di Pneumologia, Università degli Studi di Messina, Viale Gazzi

A.O.U. Policlinico, Pad. H, 98125 Messina, Italy 